\title{
Prevalence of monogenic diabetes in young adults: a community-based, cross-sectional study in Oxfordshire, UK
}

\author{
J. Kropff • M. P. Selwood • M. I. McCarthy • \\ A. J. Farmer • K. R. Owen
}

Received: 19 November 2010 / Accepted: 1 February 2011 /Published online: 25 February 2011

(C) Springer-Verlag 2011

Keywords $G C K \cdot H N F 1 A \cdot H N F 4 A \cdot M O D Y \cdot$ Monogenic beta cell disorders · Prevalence study

\section{Abbreviations \\ GADA GAD antibodies \\ LADA Latent autoimmune diabetes of adulthood GP General practitioner}

To the Editor: Recently, Shields and colleagues used cases ascertained from diagnostic genetic-testing referrals to estimate a minimum population prevalence for monogenic beta cell disorders (i.e. MODY) in the UK population [1].

J. Kropff $・$ M. I. McCarthy $\cdot$ K. R. Owen $(\bowtie)$

Oxford Centre for Diabetes,

Endocrinology and Metabolism (OCDEM), University of Oxford, Churchill Hospital,

Oxford OX3 7LJ, UK

e-mail: katharine.owen@drl.ox.ac.uk

J. Kropff • M. I. McCarthy • A. J. Farmer $\cdot$ K. R. Owen Oxford National Institute for Health Research Biomedical Research Centre, Churchill Hospital,

Oxford, UK

\section{J. Kropff}

Department of Internal Medicine, Academic Medical Centre, Amsterdam, the Netherlands

M. P. Selwood - A. J. Farmer

Department of Primary Health Care, University of Oxford, Oxford, UK

M. I. McCarthy

Wellcome Trust Centre for Human Genetics,

University of Oxford,

Oxford, UK
They estimated a minimum prevalence of 108 cases of MODY per million, with half due to mutations in HNF1AMODY. The study included all cases of MODY diagnosed through the UK testing laboratory over a 12 year period, but had the limitations of not being community-based and only included individuals who had been referred for molecular testing through secondary care.

The identification of monogenic diabetes allows targeted management and screening of at-risk relatives, so accurate prevalence figures are necessary for service provision. Despite this, information in this area is limited. This is largely because of the high costs of molecular testing, meaning that large-scale re-sequencing efforts to establish prevalence have not been done. Two studies performed prior to the availability of molecular testing estimated the prevalence of MODY in Germany at $0.14 \%$ and $1.8 \%$ of diabetes cases, respectively $[2,3]$. A paediatric survey from Germany and Austria of 40,757 individuals diagnosed with diabetes before 20 years of age identified that $0.65 \%$ had MODY confirmed by genetic testing [4]. The only previous population-based study (from Norway) reported a prevalence of 63 cases of HNF1A-MODY per million but did not investigate other genes [5].

We aimed to estimate the prevalence of diabetes subtypes in young adults diagnosed with diabetes up to age 45 years by a combination of community survey and subsequent molecular investigation [6]. The study was performed in 12 general practitioner (GP) surgeries in Oxfordshire, UK, with 118,927 individuals registered. Between March 2005 and June 2006, practice computer databases were used to identify individuals diagnosed with diabetes. The study was approved by the Oxfordshire Local Research Ethics Committee. Individuals participating in the study gave written consent. 
Figure 1 illustrates the study recruitment strategy. Participants completed a questionnaire to provide information on current medical treatment, diabetes presentation, date of diabetes diagnosis and family history. Individuals who fulfilled inclusion criteria then underwent physical examination and collection of blood for $\mathrm{HbA}_{1 \mathrm{c}}$, lipid profile, glucose, C-peptide, GAD antibodies (GADA) and DNA. Selected individuals $(n=57)$ underwent molecular testing as part of the Young Diabetes in Oxford Study [7]. $H N F 1 A$ and $H N F 4 A$ gene re-sequencing was performed in GADA-negative individuals with evidence of endogenous insulin secretion (fasting C-peptide $\geq 0.1 \mathrm{nmol} / \mathrm{l}$ ) and either diagnosed at $\leq 30$ years or $\leq 45$ years with absence of metabolic syndrome $(n=39)$. $G C K$ re-sequencing was per-

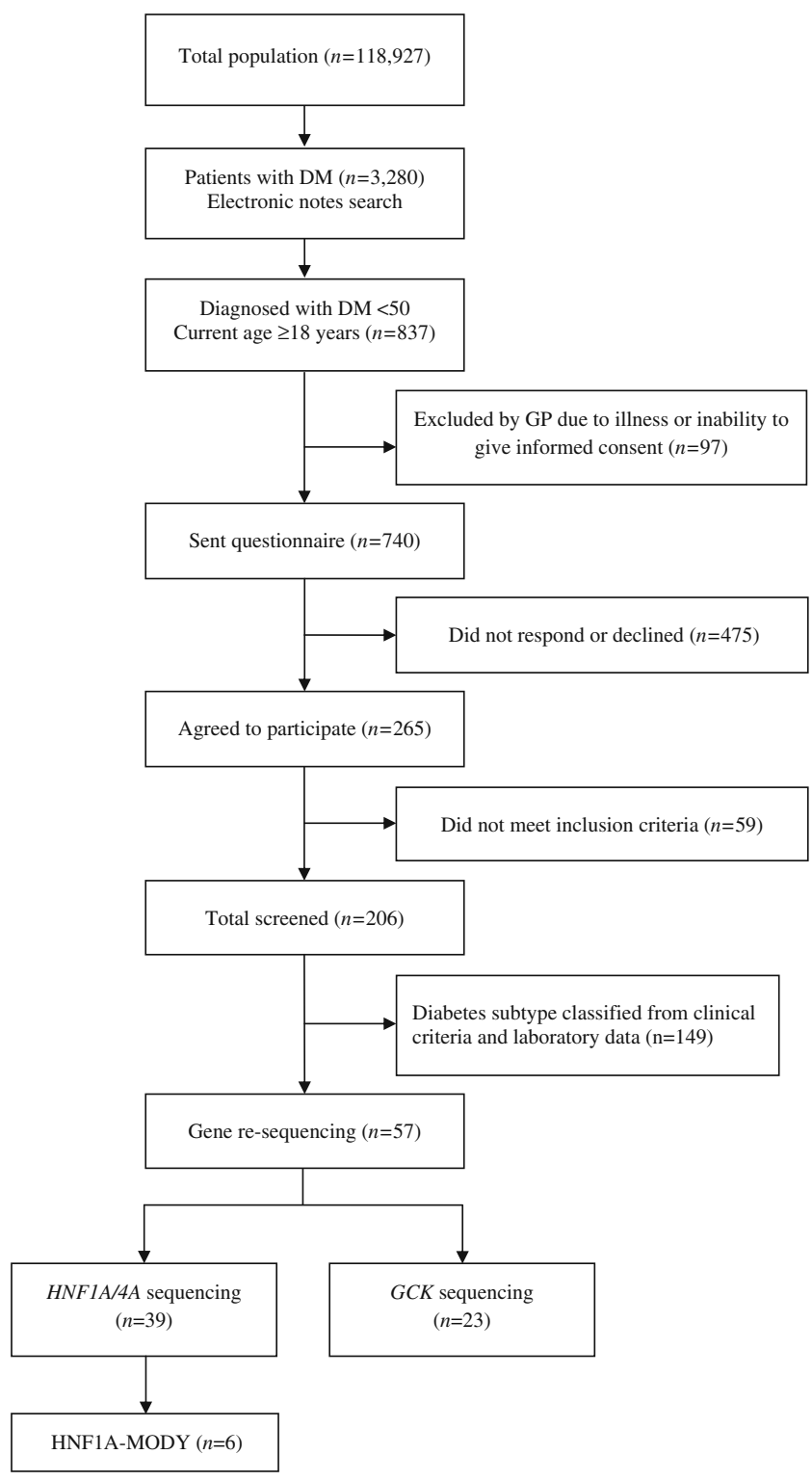

Fig. 1 Flow diagram showing the study methods and number of participants. DM, diabetes mellitus; GP, general practitioner formed in individuals with fasting C-peptide $\geq 0.1 \mathrm{nmol} / \mathrm{l}$, fasting glucose $5.5-8.0 \mathrm{mmol} / \mathrm{l}$ and $\mathrm{HbA}_{1 \mathrm{c}}<8.0 \%(n=23)$. All individuals were genotyped for a common diabetescausing mitochondrial mutation (g.3243A $>$ G) using real time PCR (Applied Biosystems Taqman 7000; Life Technologies, Carlsbad, CA, USA).

Subtypes of diabetes were defined according to clinical and laboratory data. Type 1 diabetes was defined as permanent insulin treatment within 1 year of diagnosis with evidence of severe beta cell dysfunction (C-peptide $\leq 0.09 \mathrm{nmol} / \mathrm{l}$ ), positive GADA or both. Latent autoimmune diabetes of adulthood (LADA) was defined as diabetes with positive GADA but no requirement for insulin treatment within 1 year of diagnosis. Those with evidence of endogenous insulin secretion, negative GADA and no evidence of a genetic cause were classified as type 2 diabetes. MODY mutations were classified as pathogenic if they were previously reported or co-segregated with a MODY phenotype in diabetic family members. Census population data was used to calculate the estimated UK prevalence of MODY. Statistical analysis was performed using SPSS v16 (IBM Corporation, New York, USA).

Within the 12 GP surgeries, 3,280 (2.8\%) of 118,927 individuals were identified as currently diagnosed with diabetes. Questionnaires were sent to 740 individuals (23\% of those with diabetes) identified as diagnosed with diabetes up to age 50 years and currently aged $\geq 18$ years. In total 265 responded, of whom a further 59 individuals were excluded because their age at diagnosis was $>45$ years, leaving 206 ( $28 \%$ of those invited) who attended the research clinic. There was no difference in the characteristics of responders vs non-responders. Mean $( \pm \mathrm{SD})$ age of diabetes onset was $32 \pm 12$ years and 124 participants $(60 \%)$ were male. Ethnic origin was $87 \%$ white, $8 \%$ black, $2 \%$ Asian and $3 \%$ other or mixed ethnicity.

On the basis of the criteria defined above, 69 individuals (33\%) were defined as diagnosed with type 1 diabetes and 111 (54\%) with type 2 diabetes. Twenty-six individuals (13\%) were diagnosed with other diabetes subtypes, including $18(9 \%)$ diagnosed with LADA, six (3\%) with HNF1A-MODY (from five families) and two (1\%) with mitochondrial diabetes. Other types of monogenic diabetes (GCK-MODY, HNF4A-MODY) were not found, probably because of the relative rarity of these subtypes compared with HNF1A-MODY, and also because those with GCKMODY have a phenotype of mild fasting hyperglycaemia, so may not fulfil diagnostic criteria for diabetes required for inclusion in the study. This suggests a prevalence of HNF1A-MODY of $6 / 3,280$ or $0.18 \%$ of those with diabetes, translating into a population prevalence of 50 (95\% CI 10-91) per million. Investigation of relatives of the probands identified four additional MODY mutationcarrying diabetic family members who lived locally and 
attended the same surgeries during the period of study. Taking these individuals into account led to an estimated minimum population prevalence of 84 (95\% CI 31-136) cases per million for HNF1A-MODY. This is in agreement with previous estimates from the UK and Norway [1, 5], but may represent an underestimate because we did not adjust the estimate to take into account possible cases in non-responders and the study only included individuals diagnosed before age 45 years.

This study examines the prevalence of subtypes of diabetes in primary care for the first time. We found that $13 \%$ of individuals who had been diagnosed with diabetes in young adult life had an aetiology for diabetes other than classical type 1 or type 2 diabetes. Apart from accuracy of diagnostic coding [8], this will affect their management and, for those with genetic forms of diabetes, has implications for the screening of relatives. Our study suggests there are at least 5,000 cases of HNF1A-MODY in the UK of whom nearly $90 \%$ remain unidentified [1]. We agree with Shields and colleagues that a more systematic approach to genetic investigation is required [1].

Acknowledgements The study was funded by the Oxford NIHR Biomedical Research Centre, the NIHR School for Primary Health Care Research, the European Community FP7 programme CEED3 (HEALTH-F2-2008-223211) and the Oxford Hospitals Charitable Fund. K.R. Owen is an NIHR-funded Clinician Scientist, A.J. Farmer is supported by the NIHR School of Primary Care Research.
Duality of interest J. Kropff received an unrestricted educational bursary from Novo Nordisk for elective studies. The remaining authors declare that there is no duality of interest associated with this manuscript.

\section{References}

1. Shields BM, Hicks S, Shepherd MH, Colclough K, Hattersley AT, Ellard S (2010) Maturity-onset diabetes of the young (MODY): how many cases are we missing? Diabetologia 53:2504-2508

2. Panzram G, Adolph W (1981) Heterogeneity of maturity onset diabetes at young age (MODY). Lancet 2:986

3. Ledermann HM (1995) Maturity-onset diabetes of the young (MODY) at least ten times more common in Europe than previously assumed? Diabetologia 38:1482

4. Schober E, Rami B, Grabert M et al (2009) Phenotypical aspects of maturity-onset diabetes of the young (MODY diabetes) in comparison with type 2 diabetes mellitus (T2DM) in children and adolescents: experience from a large multicentre database. Diabet Med 26:466-473

5. Eide SA, Raeder H, Johansson S et al (2008) Prevalence of HNF1A (MODY3) mutations in a Norwegian population (the HUNT2 Study). Diabet Med 25:775-781

6. Shepherd M, Sparkes AC, Hattersley AT (2001) Genetic testing in maturity onset diabetes of the young (MODY); a new challenge for the diabetic clinic. Pract Diabetes 18:16-21

7. Thanabalasingham G, Pal A, Farmer AJ, Ellard S, McCarthy MI, Owen KR (2010) Diabetologia 53(Suppl 1):S63 (abstract)

8. Stone MA, Camosso-Stefinovic J, Wilkinson J, de Lusignan S, Hattersley AT, Khunti K (2009) Incorrect and incomplete coding and classification of diabetes: a systematic review. Diabet Med 27:491-497 